\title{
The surface source model of the electrocardiogram
}

David B. Geselowitz* Howard D. Simms Jr*, Stephanie Smith*

(* Pennsylvania State University)

The bi-syncytial model of the heart leads to the result that the volume density of cardiac current source moment is proportional to the gradient of the macroscopic transmembrane action potential distribution. If the anisotropy ratios of inner and outer domains (syncytia) of the myocardium are equal, then the volume distribution of cardiac sources can be replaced by an appropriate double layer on the heart surface. For an intramyocardial electrode there is an additional term proportional to the transmembrane potential. The double layer source distribution (heart surface source model) provides a basis for calculating heart surface potentials from cardiac sources.

\section{Introduction}

The heart is a generator of electricity. Body tissues are conductors of electricity. Therefore the cardiac electric sources give rise to currents in the body and to time varying potentials in the body and on the body surface. At each observation point on the body surface there exists a time varying signal, the electrocardiogram (ECG). Conversely at each instant of time there exists a potential distribution on the body surface, the body surface potential map.

A model of the electrocardiogram may be considered to have two elements. One element consists of a description of the cardiac sources in terms of the cardiac electrophysiology. The second element consists of a relationship between these sources and the potentials they generate.

Despite its cellular nature, heart muscle electrically acts in many respects as a syncytium. One can view intracellular space as a continuous domain, or syncytium, with a resistivity which is an appropriate average of the contribution of the cell interior (cytoplasm) and that of the cell-to-cell connections (tight junctions). A second syncytium is the fluid matrix which 
forms a continuous extracellular, or interstitial, outer space. The two domains or syncytia are everywhere separated by the cell membrane. By writing the appropriate equations for the currents in the two domains, one can develop an expression which relates the cardiac sources, $\mathrm{J}^{\mathrm{i}}$, to the cellular action potential, $\phi_{\mathrm{m}}{ }^{1), 2)}$. We will refer to this approach as the bi-syncytial model $^{1,3)}$.

The torso as a volume conductor is linear, inhomogeneous, resistive, anisotropic, and bounded. It is reasonable to consider it to be divided into discrete regions such as heart, lung, bone, blood, muscle, etc. with an appropriate conductivity assigned to each region. The relationship between the cardiac sources and the potentials they generate is governed by the volume conductor problem. For bioelectric potentials the volume conductor problem has the properties of being linear, quasi-static, and obeying reciprocity ${ }^{4}$. A model of the electrocardiogram based on the bisyncytial model has been developed by Miller and Geselowitz ${ }^{1,5}$. The model appears to provide an excellent simulation of the body surface ECG for the normal heart and for a wide variety of examples of ischemia and infarction ${ }^{5)}$.

Much attention has also been devoted to the relationship between the potential distribution on the heart surface and the body surface potential distribution, with particular interest in the inverse problem of determining heart surface potentials from body surface potentials ${ }^{6}$. Thus there are parallel studies which relate body surface potentials to cardiac sources on one hand and to heart surface potentials on the other hand. Almost no work, however, has been done to relate heart surface potentials to cardiac sources.

It has been shown that under certain circumstances the distribution of cardiac sources through the volume of the myocardium can be replaced by a distribution of sources on the heart surface ${ }^{7}$. The sources are of the form of a double layer whose moment is proportional to the cellular action potential. This heart surface source provides a basis for calculating heart surface potentials from cardiac sources.

This presentation deals with the use of the bisyncytial model to characterize electric sources in anisotropic heart muscle on the basis of the bi-syncytial model. The analysis leads to the heart surface source model. The approach is macroscopic in that the currents, potentials, etc. are considered to be averages over small regions of space. Preliminary results using the heart surface source model to calculate heart surface potentials are presented.

\section{Theory}

It is possible to write down the form the equation for the potential must take. Strictly as a consequence of linearity the potential, $\mathrm{V}$, at an arbitrary point on the surface of the body (or anywhere in the volume conductor) is related to the distribution of current source dipole moment, $\mathrm{J}^{\mathrm{i}}$, throughout the heart as follows.

$$
\mathrm{V}=\int \mathrm{J}^{\mathrm{i}} \cdot \nabla \mathrm{Zdv}
$$

where $\nabla \mathrm{Z}$ is the transfer impedance function which relates the current dipole in the elemental volume $\mathrm{dv}$ to the potential it generates in the lead. From reciprocity the transfer impedance, $\nabla Z$, is equal to the lead field which is the potential in the heart resulting from the introduction 
of unit current into the lead ${ }^{8)}$. Because of the quasi-static nature of the volume conductor problem the lead field current, $\mathrm{J}_{\mathrm{L}}$ must have zero divergence ${ }^{4)}$.

$$
\nabla \cdot \mathrm{J}_{\mathrm{L}}=-\nabla \cdot \sigma \nabla \mathrm{Z}=0
$$

Finally, the bi-syncytial (bidomain) model posits that

$$
\mathrm{J}^{\mathrm{i}}=-\sigma_{\mathrm{i}} \nabla \phi_{\mathrm{m}}
$$

where $\sigma_{\mathrm{i}}$ is the conductivity of the inner or intracellular domain ${ }^{2), 4}$. If Eq. (3) is substituted in Eq. (1) we obtain the general result relating electrocardiographic potentials to the distribution of transmembrane potential throughout the heart.

$$
\mathrm{V}=-\int \sigma_{\mathrm{i}} \nabla \phi_{\mathrm{m}} \cdot \nabla \mathrm{Zdv}
$$

Through the use of vector identities it may be shown that

$$
\mathrm{V}=-\int \sigma_{\mathrm{i}} \phi_{\mathrm{m}} \nabla \mathrm{Z} \cdot \mathrm{d} \mathrm{S}_{\mathrm{H}}+\int \phi_{\mathrm{m}} \nabla \cdot \sigma_{\mathrm{i}} \nabla \mathrm{Zdv}
$$

where $\mathrm{S}_{\mathrm{H}}$ is the surface which bounds the heart ${ }^{4), 9)}$.

Heart muscle is anisotropic. The bisyncytial model considers the heart to consist of inner (intracellular) domain and an outer (interstitial) domain separated by the membrane. The conductivities of the two domains, $\sigma_{\mathrm{i}}$ and $\sigma_{\mathrm{o}}$, are each anisotropic as is the bulk conductivity, $\sigma_{\mathrm{H}}$, which is the sum of the inner and outer conductivites.

$$
\sigma_{\mathrm{H}}=\sigma_{\mathrm{i}}+\sigma_{\mathrm{o}}
$$

We will use the subscript 1 to denote the direction along the fiber axis and the subscript $t$ to denote the direction transverse to the fiber axis. A simplification results if the anisotropy ratios of the inner and outer domains are equal.

It then follows that a similar result holds for $\sigma_{\mathrm{H}}$ and that the tensor $\sigma_{\mathrm{i}}$ is proportional to the tensor $\sigma_{\mathrm{H}}$.

$$
\begin{aligned}
& \frac{\sigma_{\mathrm{it}}}{\sigma_{\mathrm{il}}}=\frac{\sigma_{\mathrm{ot}}}{\sigma_{\mathrm{ol}}}=\beta \\
& \frac{\sigma_{\mathrm{it}}}{\sigma_{\mathrm{Ht}}}=\frac{\sigma_{\mathrm{il}}}{\sigma_{\mathrm{Hi}}} \equiv \alpha
\end{aligned}
$$

From Eq. (2) the second term on the right of Eq. (5) then vanishes, and

$$
\mathrm{V}=-\int \sigma_{\mathrm{i}} \phi_{\mathrm{m}} \nabla \mathrm{Z} \cdot \mathrm{d} \mathrm{S}_{\mathrm{H}}
$$

In this case the volume distribution of sources in the heart can be replaced by a dipole layer on the heart surface whose moment is proportional to the transmembrane action potential.

The result of Eq. (9) must be modified if the electrode is in the heart. Consider a lead with an intramyocardial electrode and a distant reference electrode. Then

$$
\mathrm{V}=-\int \sigma_{\mathrm{i}} \phi_{\mathrm{m}} \nabla \mathrm{Z} \cdot \mathrm{d} \mathrm{S}_{\mathrm{H}}+\alpha \int \phi_{\mathrm{m}} \nabla \cdot \sigma_{\mathrm{H}} \nabla \mathrm{Zdv}
$$

In this case the lead field is associated with injection of unit current into the myocardial electrode and its removal at the distant electrode. The second integral on the right involves the divergence of the lead field current which now vanishes everywhere except at the 
electrode, or observation point, where it becomes infinite. Under this circumstance

$$
\mathrm{V}=-\int \sigma_{\mathrm{i}} \phi_{\mathrm{m}} \nabla \mathrm{Z} \cdot \mathrm{d} \mathrm{S}_{\mathrm{H}}-\alpha \phi_{\mathrm{m}}
$$

Eq. (9) can be considered a special case of Eq. (11) for a lead outside the heart.

\section{Heart surface potentials}

Eq. (4) forms the basis of the Miller-Geselowitz model for simulating the body surface electrocardiogram. This equation, however, is not suitable for calculating heart surface potentials. Eq. (9), on the othe hand is more appropriate since the sources are of the form of a double layer on the heart surface. The key characteristic of the double layer that makes it suitable for performing the calculation is that the potential is finite as one approaches a point on the surface.

For simplicity in calculating heart surface potentials we assume that the torso is homogeneous with conductivity, $\sigma=\sigma_{\mathrm{H}}$, and that the effect of the torso boundary can be ignored. In this case

$$
\nabla Z=\frac{1}{4 \pi \sigma \nabla}\left(\frac{1}{r}\right)
$$

where $r$ is the distance from a point on the heart surface to the observation point. Hence, if $\sigma_{\mathrm{i}}$ is taken to be constant in the heart,

$$
\mathrm{V}=\frac{-\sigma_{\mathrm{i}}}{4 \pi \sigma} \int \phi_{\mathrm{m}} \nabla\left(\frac{1}{r}\right) \cdot \mathrm{dS}_{\mathrm{H}}
$$

Note that the heart surface is the complete surface enclosing the active region of the heart, and includes the endcardium and the epicardium.

Eps. (4), (9), and (13) require a description of the transmembrane action potential, $\phi_{\mathrm{m}}$, throughout the myocardium or on the heart surface. The time course of $\phi_{\mathrm{m}}$ at any point in the heart can be determined if the waveshape is known, and the activation time is known. For the normal heart the evidence is that the action potential waveshapes are similar except for a progressive shortening of the action potential duration from endocardium to epicardium. In the case of the normal heart, the upstroke of the action potential can be considered a step function of constant amplitude. Hence the surface sources are either "off" in the resting state, or" on" when activated. The boundary between these two states is the activation isochrone, and QRS is completely determined by the activation isochrones for the normal heart.

To model ischemia, action potentials and conduction velocity are modified in the region of injury on the basis of available electrophysiologic data. As ischemia progresses there are characteristic changes in the action potentials, including a decrease in the magnitude of the resting potential, a decrease in action potential amplitude, a shortening or prolongation of the action potential, and an increase in the risetime of the upstroke, or depolarization phase. A cell may eventually become unresposive and finally die.

The surface source model, Eq. (13), was used to calculate epicardial potentials for a number of cases $^{10)}$. Action potential data were taken from Miller and Geselowitz ${ }^{1,5)}$. Details of the numerical procedures are contained in the Masters thesis by Simms ${ }^{10)}$. 


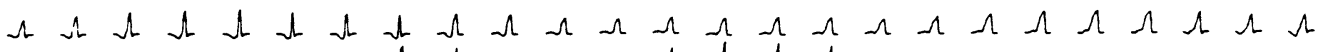

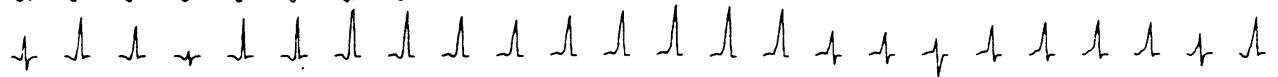

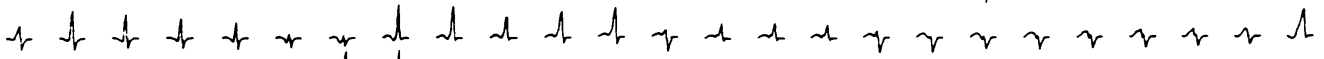

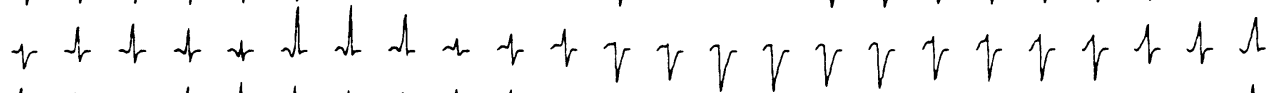

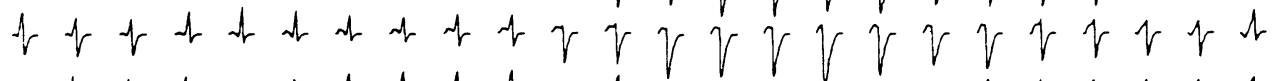

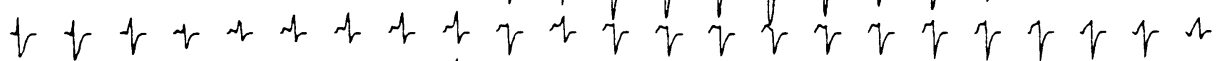

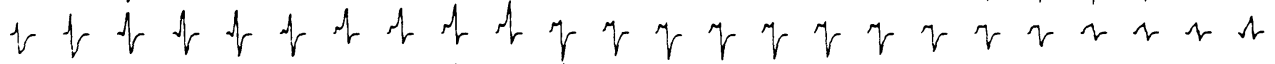

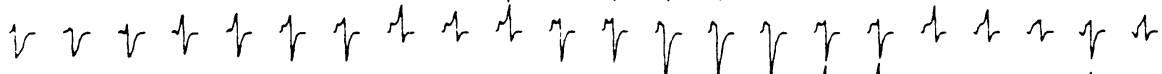

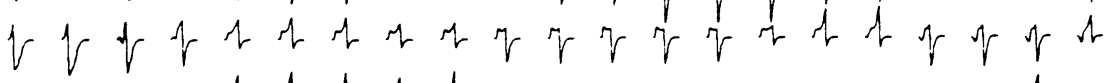

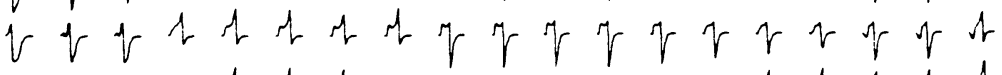

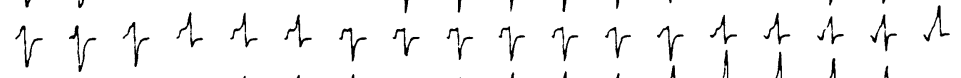

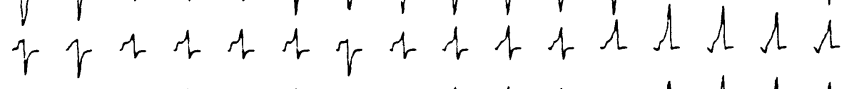

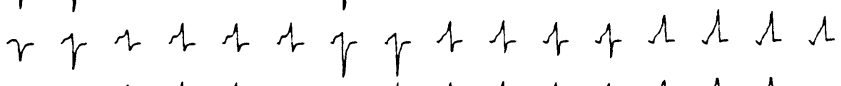

$$
\begin{aligned}
& \text { is if } p \text { is t it of } \Lambda \Lambda \Lambda
\end{aligned}
$$

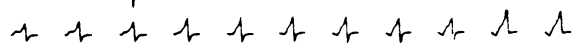

$$
\begin{aligned}
& \text { of if of of of of o }
\end{aligned}
$$

Figure 1 Distribution of computed unipolar electrograms over the anterior surface of the ventricles for the normal heart. The heart is placed in an unbounded homogeneous conducting medium. Electrograms are computed using the Miller-Geselowitz surface source model.

Following are results of simulations of electrograms over the anterior surface of the ventricles during activation ${ }^{10}$. Figure 1 shows results for the normal heart. The simulated electrograms may be compared with electrograms on the anterior surface of a canine heart reported by Arisi et al. ${ }^{11}$. There is considerable agreement in waveshapes. The electrograsms recorded by Arisi are about three times larger in amplitude than our simulated electrograms. We attribute this difference to the fact that the simulation assumes the heart is surrounded by conducting tissues, where as Arisi obtained his recordings on the exposed heart. Figure 2 shows a simulation of a transmural injury reported previously ${ }^{5}$. Note the pattern of QS waves over the injury.

\section{Discussion}

The bi-syncytial model is a macroscopic one in that current densities, potentials, and conductivities are averages over regions containing many cells. At the microscopic level, extracellular potentials close to the cells reflect the cellular nature of the tissue, but this effect becomes smaller at larger distances ${ }^{2}$.

Eq. (4) follows from the bi-syncytial model and is the basis of the model of Miller and Geselowitz. At each instant during the cardiac cycle, the ECG depends on the spatial distribution of the cardiac action potential throughout the heart, and more particularly on the spatial gradien (derivative) of $\phi_{\mathrm{m}}$. Eq. (4) also involves the transfer coefficients, $\nabla Z$, which are 


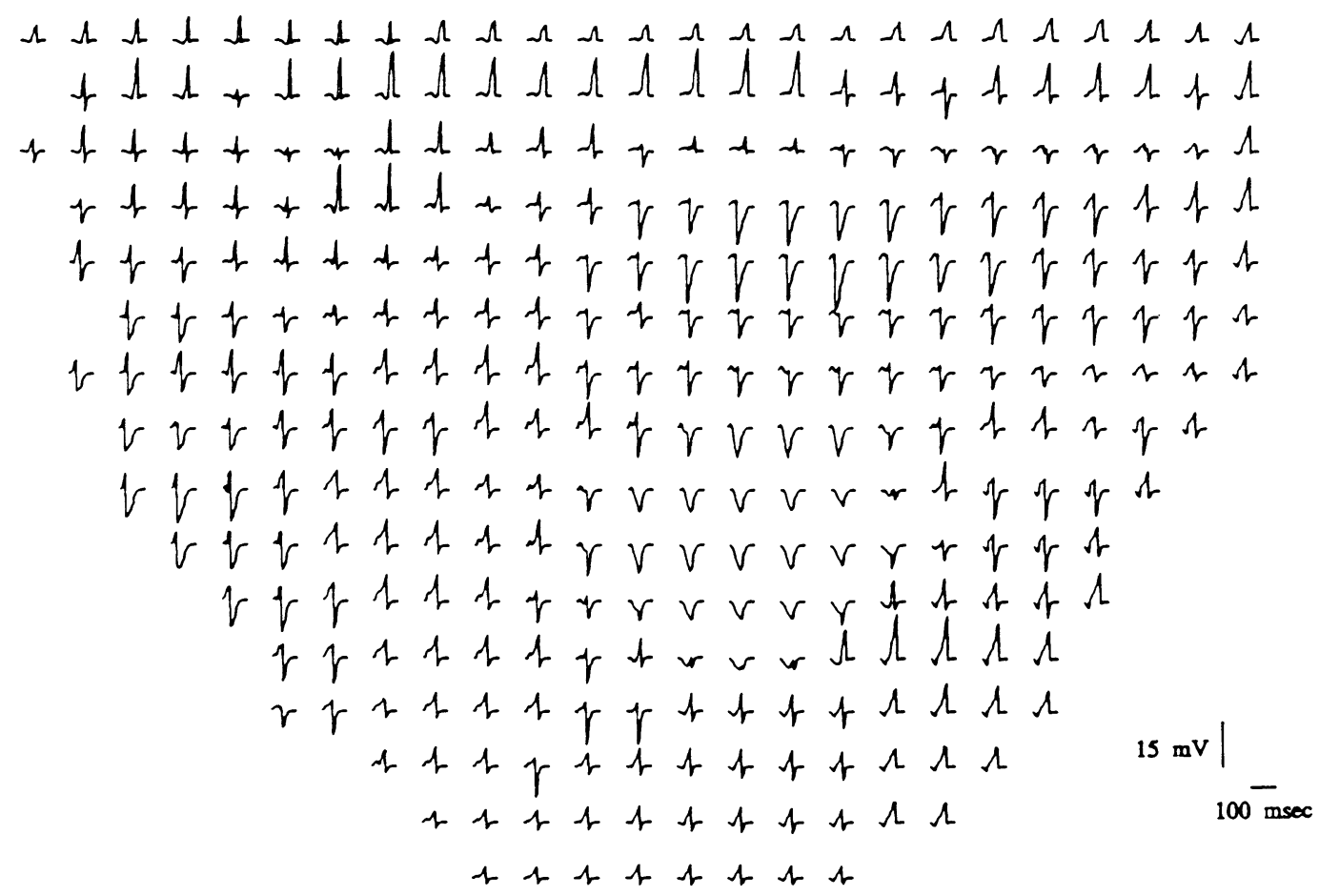

Figure 2 Distribution of computed unipolar electrograms over the anterior surface of the ventricles for a transmural injury in the anterior portion of the left ventricle. The heart is placed in an unbounded homogeneous conducting medium. Electrograms are computed using the MillerGeselowitz surface source model with the injury adapted from ${ }^{[5]}$. Electrograms over the region of injury are chracterized by smooth QS waves.

generated by a digital computer solution of the volume conductor problem.

Eq. (11) is the basic result of the surface source model. It is valid for the special case of equal anisotropy ratios. The distribution of sources throughout the heart is replaced by a double layer on the heart surface where the moment of the double layer is proportional to the transmembrane potential. Other aspects of the volume conductor are incorporated in $\nabla \mathrm{Z}$. If the volume conductor is taken to be homogeneous and unbounded, then Eq. (11) reduces to Eq. (13). We have shown that the effect of the torso boundary is small for calculating epicardial electrograms ${ }^{10)}$.

For an intramyocardial electrode there is an additional term proportional to the membrane potential at the observation point. In the one dimensional case this result follows from the linear core conductor model (cable theory). The present derivation does not call no cable theory per se, although the bidomain model can be considered a three dimensional extrapolation of cable theory.

Cuppen and van Oosterom were led to the surface source model by considering that during activation the cardiac source distribution is a uniform double layer and that the heart is isotropic $^{13)}$. The derivation presented here is a more general one that does not require a uniform double layer in an isotropic conducting medium, and that applies to recovery as well as excitation. Van Oosterom and coworkers have reported success in applying this model to 
a solution of the inverse problem for activation of the normal heart ${ }^{13), 14}$. Since Eq. (9) follows from the assumption of equal anisotropy ratios, their results can be taken to suggest that the volume integral in Eq. (5) may be ignored even though the anisotropy ratios in the myocardium are not necessarily equal. In applying the surface source model to cases of injury to the heart the simplification that during activation the sources are either on or off is no longer valid, and modifications in their approach to the inverse problem may be necessary.

A key quality of an appropriate model for the electrocardiogram is that it allows us to determine the genesis of features of the waveform. Previous application of the bi-syncytial model to the simulation of body surface electrograms with the aid of the Miller-Geselowitz model gave good results for simulated body surface electrocardiograms, including acute ischemia and infarction. Our newer results indicate that the model also predicts heart surface electrograms. In the latter case the volume distribution of sources is replaced by the surface sources. The surface source model appears to provide good simulations of heart surface electrograms, and bridges the gap between approaches to the body surface electrocardiogram predicated on cardiac sources and those predicated on heart surface potentials.

\section{Acknowledgement}

This work was supported by a grant HL33715 from the National Institutes of Health.

\section{References}

1) Miller WT III, Geselowitz DB : Simulation studies of the electrocardiogram. I . The normal heart. Circ Res $43: 301 \sim 315,1978$

2) Geselowitz DB, Miller WT III : A bidomain model for anisotropic cardiac muscle. Ann Biomed Engr 11 : 191 206, 1983

3) Tung L: A bidomain model for describing ischemic myocardial $d^{-} \mathrm{c}$ potentials. Ph D, thesis, MIT, Cambridge, MA, 1978

4) Geselowitz DB : On the theory of the electrocardiogram. Proc IEEE $77: 857 \sim 876,1989$

5 ) Miller WT, Geselowitz DB : Simulation studies of the electrocardiogram. II. Ischemia and infarction. Circ Res $43: 315 \sim 323,1978$

6 ) Barr RC, Ramsey M III, Spach MS : Relating epicardial to body surface potential distributions by means of transfer coefficients based on geometry measurements. IEEE Trans Biomed Eng BME-24:1 11, 1977

7) Yamashita Y, Geselowitz DB : Source-field relationships for cardiac generators on the heart surface based on their trasfer coefficients. IEEE Trans Biomed Eng BME-32: 964 970, 1985

8 ) McFee R, Johnston FD : Electrocardiographic leads : III, Synthesis. Circulation 9:868 880, 1954

9) Geselowitz DB : Use of time integrals of the ECG to solve the inverse problem. IEEE Trans Biomed Eng BME-32: 73 75, 1985

10) Simms HD : Calculation of heart surface potentials using a surface source model with consideration of myocardial injury. M.S. Thesis in Bioengineering, Pennsylvania State University, 1992

11) Arisi G, Macchi E, Baruffi S, Spaggiari S, Taccardi B : Potential fields on the ventricular surface of the exposed dog heart during normal excitation. Circulation Research $52: 706 \sim 715,1983$

12) Diaz PJ, Rudy $\mathrm{Y}$, Plonsey R : Intercalated discs as a cause for discontinuous propagation in cardiac muscle: A theoretical sumulation. Ann Biomed Eng $11: 177 \sim 189,1983$

13) Cuppen JJM, van Oosterom A : Model studies with the inversely calculated isochrones of ventricular depolarization. IEEE Trans Biomed Eng BME-31:652 659, 1984

14) Huiskamp G, van Oosterom A : The depolarization sequence of the human heart surface computed from measured body surface potentials. IEEE Trans Biomed Eng. BME-35: 1047 1058, 1988 